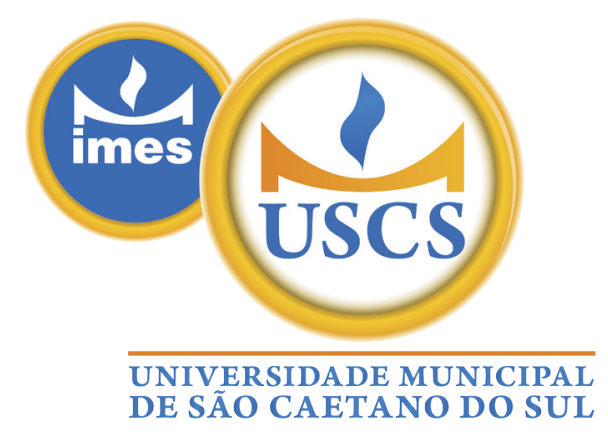

Publicação do Programa de Pós-graduação em Comunicação Social da Universidade Municipal de São Caetano do Sul

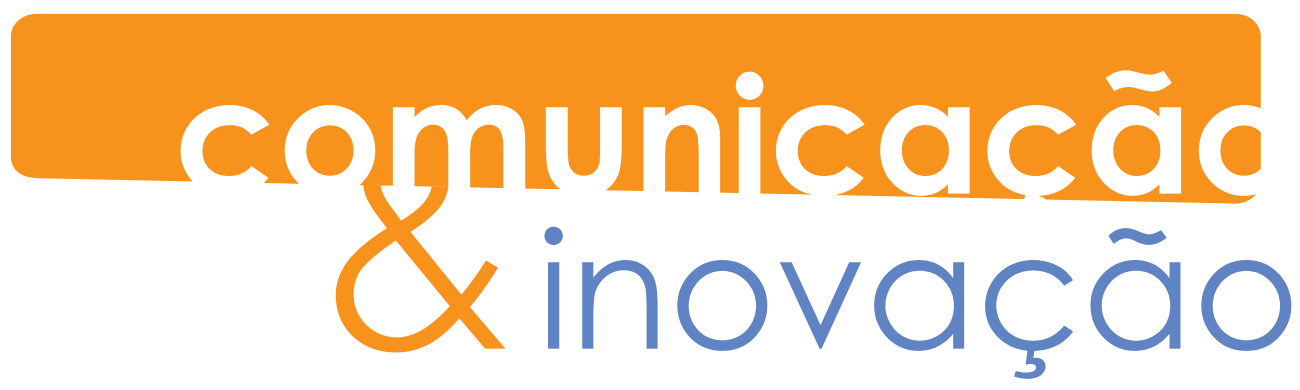

volume 15 • número 29 • julho - dezembro 2014 


\section{communicacãd \\ \&inovação}

Integrada à RED - Iberoamericana de Revistas de Comunicação e Cultura

(www.revistadecomunication.org)

\section{Revistas integrantes da RED}

Actas y Comunicaciones del Instituto de Historia Antigua y Medieval

Anagramas

Anales de Historia Antigua, Medieval y Moderna

Anàlisi

Anuario de Investigaciones

Anuario ININCO

Anuario UNESCO/UMESP de Comunicación

Regional

Aportes de la Comunicación y la Cultura

Arandu

Arte Críticas

Boletín Científico Sapiens Research

C+I Comunicación e Información

Chasqui

Comunicação \& Artes

Comunicação \& Educação

Comunicação \& Inovação

Comunicação \& Sociedade

Comunicação Pro

COMUNICACIÓN U.P.B.

Comunicación y Hombre

Comunicación y Medios

Comunicación y Sociedad (México)

Comunicación y Sociedad (España)

Comunicación. Estudios Venezolanos de la Comunicación

Comunicar. Revista Científica Iberoamericana de

Comunicación y

Educación

Conexiones. Revista Iberoamericana de Comunicación

\author{
Constelaciones de la Comunicación \\ Contratexto \\ Convergencia. Revista de Ciencias Sociales \\ Criterio Libre \\ Cuaderno de H Ideas \\ Cuadernos de información \\ Dedica. Revista de Educação e Humanidades \\ Diálogos de la Comunicación \\ Disertaciones \\ Doxa.comunicación. Revista interdisciplinar de \\ comunicación y ciencias \\ sociales \\ Em Questão \\ Escribania \\ Espacios Públicos \\ Estudios de Periodismo y Relaciones Públicas \\ Estudios sobre las Culturas Contemporáneas \\ Exedra. Revista Científica \\ Extensión en Red \\ Figuraciones \\ Global Media Journal \\ Icono 14 \\ Imagofagia \\ In-mediaciones de la Comunicación \\ INSIGHTS \\ Interação \\ Interacción. Revista de Comunicación Educativa \\ Intercom: Revista Brasileira de Ciências da Co- \\ municação \\ Interlenguajes \\ Intersecciones \\ Isla Flotante
}

Dados INTERNACIONAIS de CATALOGaÇão Na PUbLICAÇão (CIP)

Comunicação \& Inovação / [Publicação do] Curso de Comunicação Social da Universidade Municipal de São Caetano do Sul - v. 6, n. 11 jul/dez 2005 - São Caetano do Sul : Universidade IMES, 2005

Semestral

Resumo em inglês e português

Continuação da Revista IMES Comunicação, v. 1, n. 1-10, 2000-2005

ISSN 2178-0145

1. Comunicação - Periódico. I. Universidade Municipal de São Caetano do Sul 


\section{comunicacãd \\ \&inovação}

\section{Revistas integrantes da RED}

Izquierdas

La Trama de la Comunicación

Líbero

LIS. Letra. Imagen. Sonido. La Ciudad Mediatizada

Luciérnaga

Lumina

Más Poder Local

Mediaciones

Medios y Enteros

MiRatón

Newsletter

Oficios Terrestres

Ojos de Buey

Ojo de Buey. Revista digital de cine

Palabra Clave

Papeles de trabajo

Pedagogía Social. Revista Interuniversitaria

Perspectivas de la Comunicación

Pixel-Bit. Revista de Medios y Educación

Poliantea

Prisma Social

Punto Cero

Question

Quórum Académico

Razón y Palabra

Revista Científica Digital Pensamiento Comunicacional Latinoamericano
Revista de Educación Inclusiva

Revista de la Facultad de Derecho y Ciencias

Sociales

Revista Electrónica de Investigación y Docencia (REID)

Revista Electrónica Nodos de la Comunicación REVISTA FAMECOS: mídia, cultura e tecnologia

Revista Interamericana de Nuevas Tecnologías de la Información

Revista Internacional de Folkcomunicação - Revista Folkcom (on line)

Revista latina de Comunicación Social

Revista Mexicana de Comunicación

RIALAI

RIED. Revista Iberoamericana de Educación a

Distancia

Signo y Pensamiento

Sinergia

Tecnología y Comunicación Educativas

Telos. Cuadernos de Comunicación e Innovación

Temas de Comunicación

Temas y Problemas de Comunicación

Trama

Tram[p]as de la Comunicación y la Cultura

Ventana Indiscreta

Versión. Estudios de Comunicación y Política 


\title{
communicacãd dinovação
}

\author{
volume 15 • número 29 \\ julho - dezembro 2014
}

Fechamento desta edição: outubro 2014

Reitor

Marcos Sidnei Bassi

Pró-Reitor Administrativo e Financeiro

Gilberto da Silva Alves

\section{Pró-Reitor de Graduação}

Marco Antonio Biffi

Pró-Reitor de Pós-Graduação e Pesquisa

Maria do Carmo Romeiro

Gestor do Programa de Pós-Graduação

Stricto Sensu em Comunicação

Herom Vargas Silva

\section{Editora}

Regina Rosetti

\section{Gestor de Comissão de Publicações}

Acadêmicas

Marco Antonio Pinheiro da Silveira

\section{Jornalista Responsável}

Roberto Elísio dos Santos - MTb 15.637

\section{Comissão Editorial}

Dr. Gino Giacomini Filho

Dr. Roberto Elísio dos Santos

Dra. Regina Rossetti

\section{Conselho Consultivo}

Ángel Badillo Matos, USAL, Espanha

Ana Silvia Lopes Davi Médola, UNESP,

Brasil

Dilma de Melo Silva, ECA/USP, Brasil

Eron Brum, Uniderp, Brasil

Fernando Andacht, University of

Ottawa, Canadá

Gilson Monteiro, UFAM, Brasil

Henrique Paiva de Magalhaes, UFPB, Brasil

Jean Louis Santorio, ENCPB, França

John Lent, School of Communications and Theatre Temple University, Estados Unidos da América do Norte
José Antonio Moreiro Gonzalez, Universidad Carlos III/Madri, Espanha Juan Pablo Gonzalez, UC, Chile Juremir Machado da Silva, PUC/RS, Brasil Kleber Santos de Mendonça, UFF, Brasil Laura Vazquez Hutnik, UBA, Argentina Liana Gottlieb, Casper Líbero, Brasil Maria Amélia Chagas Gaiarsa, UCSAL, Brasil

Maria Ataide Malcher, UFPA, Brasil

Maria das Graças Andrade Ataide de

Almeida, UFRPE, Brasil

Maria das Graças Targino, UFPI, Brasil

Maria Érica de Oliveira Lima, UFRN, Brasil

Maria Lucia Santaella Braga, PUC/SP, Brasil

Marcius Freire, Unicamp, Brasil

Marita Fornaro, Universidad de la

República, Uruguai

Nello Barile, IULM, Itália

Neusa Demartini Gomes, PUC/RS, Brasil

Nilda Aparecida Jacks, UFRGS, Brasil

Paulo Eduardo Ramos, UNIFESP, Brasil

Sandra Lucia Amaral de Assis Reimão,

ECA/USP, Brasil

Sergio Dayrell Porto, UNB, Brasil

Tanius Karam, UNAM, México

Winfried Maximilian Nöth, PUC/SP, Alemanha

Revisão e Editoração

Tikinet

\section{Comunicação \& Inovação}

Rua Santo Antônio, 50 - Centro

São Caetano do Sul - SP

Tel.: (11) 4239-3200 (ramal 3441)

E-mail: rrossetti@uscs.edu.br

Página na Web: http://seer.uscs.edu.br/index.

php/revista_comunicacao_inovacao

A Universidade Municipal de São Caetano do Sul, em seus cadernos e revistas, respeita a liberdade intelectual dos autores, publica os originais que lhe são entregues, sem com isso concordar necessariamente com as opiniões expressas. 


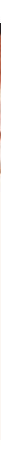

\section{Dossiê Comunicação e nOVAS tecnologias}

Análise visual do Twitter

Elias Estevão Goulart

Sidney Fels

COMUNICAÇÃO, ARTE E TECNOLOGIAS: TRÊS MOMENTOS DA CRIAÇ̃̃o E APROPRIAÇ̃̃o CONTEMPORÂNEAS

Míriam Cristina Carlos Silva

Paulo Celso da Silva

DOCUMENTÁRIO E CONTEMPORANEIDADE: UMA REFLEXÃO SOBRE

VÍDEOS PRODUZIDOS POR TELEFONES CELULARES

Ana Paula Oliveira

Características E habilidades no ambiente digital: a CUltura participativa sob os aportes de Jenkins e Murray

Alan César Belo Angeluci

As REDES SOCIAIS COMO FERRAMENTAS de COMUNICAÇÃo MERCADOLÓGICA PARA SEGMENTOS DE PÚBLICOS REGIONAIS: UM CASO DE PRODUTOS DE BENS DE CONSUMO

Monica Franchi Carniello

Gustavo Ribeiro Rosa

Artigos

A PESQUisa em Mídia e RELigião no Brasil: arTiculações teóricas NA FORMAÇ̃̃o DE UMA ÁREA DE ESTUdOS

Luis Mauro Sa Martino

NARRATIVAS DE EMPREENDEDORES SOCIAIS PORTUGUESES E BRASILEIROS: PERCURSOS DE VIDA E PROJETOS DE TRANSFORMAÇ̃̃o Vander Casaqui

Hospício Cultural: construindo PONTES ENTRE TeORIA E PRÁtica NO CAMPO DA COMUNICAÇão

Telma Johnson 
Honra ao mérito: como a instituição do Prêmio Esso CONTRIBUIU PARA A ADOÇÃo DOS REFERENCIAIS DE OBJETIVIDADE JORNALÍSTICA NO BRASIL

Robson Dias

MÉTOdos de CRIATIVIDAde PARA GERIR A COMUNICAÇão

Felipe Chibás Ortiz

O Caráter multidisciplinar da Comunicação Visual em HOSPITAIS

Mônica de Moraes Oliveira

Sandra Maria Ribeiro de Souza

AbrangênCias locais do Jornalismo online do Tocantins

Liana Vidigal Rocha

Sérgio Ricardo Soares

Valmir Teixeira Araújo

RESENHAS

As DESCOBERTAS DA MEDICINA No SÉCULO XX

Carlos Eduardo de Magalhães Barreto (in memorian)

UM SOMBRIO CENÁRIO PROMISSOR

Katarini Miguel

TransPosições E hibridizaÇões eMPREgadas EM PRODUTOS MIDIÁTICOS INOVADORES

David Santoro Junior

Dicionário de Comunicação Publicitária

Aguinaldo Ricciotti Pettinati 